\title{
Predicting loss reserves using quantile regression Running title: Quantile regression loss reserve models
}

\author{
Chan, J. S .K. ${ }^{1 *}$ \\ ${ }^{1}$ Department of Mathematics and Statistics, Sydney University
}

\begin{abstract}
Traditional loss reserves models focus on the mean of the conditional loss distribution. If the factors driving high claims differ systematically from those driving medium to low claims, alternative models that differentiate such differences are required. We propose quantile regression model loss reserving as the model offers potentially different solutions at distinct quantiles so that the effects of risk factors are differentiated at different points of the conditional loss distribution. Due to its nonparametric nature, quantile regression is free of the model assumptions for traditional mean regression models, including homogeneous variance across risk factors and symmetric and light tails, etc. These model assumptions have posed a great barrier in applications as they are often not met in the claim data. Using two sets of run-off triangle claim data from Israel and Queensland, Australia, we present the quantile regression approach that illustrates the sensitivity of claim size to risk factors, namely the trend pattern and initial claim level, in different quantiles. Trained models are applied to predict future claims in the lower run-off triangle. Findings suggest that reliance on standard loss reserves techniques gives rise to misleading inferences and that claim size is not homogeneously driven by the same risk factors across quantiles.
\end{abstract}

Key words: Quantile regression, loss reserves, run-off triangle, risk heterogeneity, extreme outlier.

\section{Introduction}

An insurance company promises to pay claims to the insureds if some defined events (injury, accident, death, etc.) occur. However in many cases, claims originating in a particular year are often settled with a time delay of years or perhaps decades. Therefore, a method to estimate the expected liability is needed so that the insurer can calculate the profit of written policies, and allocate reserved assets to ensure liquidity. Since loss reserves generally represent by far the largest liability, and the greatest source of financial uncertainty in an insurance company, an appropriate valuation of insurance liabilities including risk margin is one of the most important

\footnotetext{
${ }^{*}$ Corresponding author.
} 
issues for a general insurer. Risk margin is the component of the value of claims liability that relates to the inherent uncertainty.

The significance of providing appropriate valuation of insurance liabilities is well understood by the actuarial profession and has been debated by both practitioners and academic actuaries alike. Specifically, the aim is to develop statistical models, the loss reserve models to analyse loss reserves data in the format of a run-off triangle and predict future claims in the lower triangle. A run-off triangle is a matrix where each row corresponds to the year of an accident (the so-called policy/accident year), and each column corresponds to the number of years between the accident year, and the year in which the claim was made (the so-called development/lag year). Let $Y_{i, j}$ denote the value of claims paid by an insurance company in policy year $i$, and settled after $j-1$ years (or lag year $j$ ). The observation $Y_{i, j}, i=$ $1, \ldots, n ; j \leq n-i+1$, over a period of $n$ policy years can be presented by a run-off triangle in Figure 1.

\begin{tabular}{|c|c|c|c|c|c|c|c|c|c|}
\hline & \multicolumn{8}{|c|}{ Lag year $\mathbf{j}$} \\
\hline & & 1 & 2 & 3 & . & . & . & $n-1$ & $\mathrm{n}$ \\
\hline \multirow{8}{*}{$\begin{array}{c}\text { Policy } \\
\text { year } \\
\text { i }\end{array}$} & 1 & $Y_{1,1}$ & $Y_{1,2}$ & $Y_{1,3}$ & . & . & . & $Y_{1, n-1}$ & $Y_{1, n}$ \\
\hline & 2 & $Y_{2,1}$ & $Y_{2,2}$ & . & . & . & . & $Y_{2, n-1}$ & \\
\hline & 3 & $Y_{3,1}$ & . & . & . & . & . & & \\
\hline & . & . & . & . & . & . & 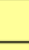 & & \\
\hline &. & . & . & . & . & & & & \\
\hline & . & . & . & . & & & & & \\
\hline & $\mathrm{n}-1$ & $Y_{n-1,1}$ & $Y_{n-1,2}$ & & & & & & \\
\hline & $\mathrm{n}$ & $Y_{n, 1}$ & & & & & & & \\
\hline
\end{tabular}

Figure 1: Run-off triangle for loss reserves data

Using the $T_{u}=n(n+1) / 2$ observed claims in the upper triangle, we aim to predict the $T_{l}=n(n-1) / 2$ future claims in the lower triangle. The values in each diagonal correspond to claims in one single calendar year.

There have been several approaches considered which range from those that involve little analysis of the underlying claim portfolio to those that involve significant analysis of the uncertainty using a wide range of information and techniques, including sophisticated stochastic models (Taylor [22] and Klugman [12]). Traditional models using the generalized linear model approach (de Jone and Heller [8]) in the stochastic framework are based on loss distributions which are estimated using historical data and the claims liability is evaluated using central estimate which is typically defined as the expected value over the entire loss distribution. However these models have implicit assumptions of risk homogeneity which refers to homogeneous loss distribution across risk factors and absence of catastrophic losses. With the inherent uncertainty that may arise, the mean estimator is not statistically robust and therefore sensitive to outlier claims. Hence claims liability measures often differ from their central estimates. 
In practice, the approach adopted is typically to then set an insurance provision so that, to a specified probability say $75 \%$, the provision will eventually be sufficient to cover the run-off claims. When this margin is then added to the central estimate, it should provide a reasonable valuation of claims liability and therefore increases the likelihood of providing sufficient provision to meet the claims liability. Moreover actuaries are more concerned with high claims due to their possibly adverse impact on the insurance fund. In this regard, it is worth noting that the more volatile a portfolios runoffs or those that display heavy tailed features may require a higher risk margin, since the potential for large swings in reserves is greater than that of a more stable portfolio.

To address these issues, percentile or quantile methods is most prevalent in practice and this provides a good foundation or the quantile regression models we consider. The quantile regression is proposed by Koenker and Bassett [13] and popularized, in part, by Buchinsky [4] and Koenker and Hallock [14] for the advancement of loss reserves methodology. The quantile of a distribution for a random variable $\mathrm{Y}$ is defined as $y_{\tau}=\inf \left\{y: F_{Y}(y) \geq \tau\right\}$ where $1-$ $\tau$ is the probability of ruin in actuarial studies.

The quantile regression model has several advantages over the traditional loss reserves models. Firstly, it differentiates risk factors that drive high level claims from those which drive low level claims. It is, therefore, possible to determine if loses are homogeneously driven by the same determinants and to distinguish risk factors impacting resolution costs of expensive loses from the factors impacting less expensive loses. Hence quantile regression loss reserve models analyse risk factors at all points of the distribution particularly the upper tails for expensive loses instead of purely the center.

Secondly, quantile regression is free from some disadvantages of the traditional models: omitted variables bias, heteroskedasticity and non-normal error distributions, all of which prevail in the loss reserves data. Omitted variables bias refers to the bias in the outcome variable when there are many other unmeasured factors that are not included in the mean of data distribution. Hence the outcomes cannot change by more than some upper limit set by the measured factors, but may change by less when other unmeasured factors are limiting (Cade and Noon [5]). In loss reserves model, failure to include all relevant variables often occurs because of insufficient knowledge of the many underlying risk factors that drive the claim process or the inability to measure all relevant processes. This is particularly the case when aggregate instead of individual claims are modelled. This omitted variables bias are allowed for in different levels of quantile regression.

Thirdly, quantile regression requires no specification of how variance changes are linked to the mean and hence it can be applied to model heterogeneous variation in loss distribution. In loss reserves model, heteroskedasticity caused by extreme claims often results in inflated variance estimates, leading to contaminated parameter estimates in the mean of the loss distribution. In quantile regression, effects of outliers appear only in the higher quantiles on the two ends as they adopt heavier weights only in the loss function of higher quantile. Thus, quantile regression is robust to the presence of outliers. 
Lastly, most traditional models assume Gaussian errors within the generalized linear model framework. Others consider errors in the exponential family. Chan, Choy and Makov [7] proposed the generalized- $t$ (GT) distribution which contains several important families of distributions including the Student- $t$, exponential power and uniform distributions for the log of claim sizes. However they remarked that as the log-linear model is more sensitive to low values than large values, the residuals in the empirical study are negatively skewed and that one should consider some skewed error distributions. While the GT distribution is sophisticated and general but still inappropriate to allow for skewed errors after logarithmic transformation, the quantile regression is perhaps a simple and yet more efficient alternative when the error distribution is nonnormal (Buchinsky,1998) as the quantile regression avoids this distribution assumption altogether. In summary, quantile regression provides a way of understanding and testing how the relationships between claims and other risk factors change across the distribution of conditional claims and it avoids the distribution assumptions in mean regression.

Although the median and quantile regression have not been used as extensively as the mean regression, using the ordinary least square (OLS) method in particular, in the empirical literature, quantile regression has been applied in diverse fields including Buchinsky [2] , [3], [4] on labor economics, Eide and Showalter [9] on earnings mobility, Cade and Noon [5] and Cade, Terrell and Schroeder [6] on ecology and Eide and Showalter (1998) on education, etc. Financial applications include Barnes and Hughes [1] and Engle and Manganelli [10] in Value at Risk estimation. Quantile regression in insurance applications can be found in Portnoy [21] for the graduation of mortality table rates, Pitt [20] for the claim termination rates for income protection insurance and Kudryavtsev [19] for rate-making in heterogeneous insurance portfolios. However none of these works focus on loss reserve models for run-off triangle using the trend of claims to predict future claims. This paper aims to pioneer the application in this area.

The paper is organized as follows. In Section 2, the theory of quantile regression is presented. Section 3 describes two empirical examples in which quantile regression is applied to the loss reserves data presented in a run-off triangle. Trends of loss across lag years are identified at different quantile levels. Section 4 predicts future claims using the trained models and assesses the predicted total future claims by comparison with those using the chain ladder (CL) method and the model of Chan, Choy and Makov [7] with GT distribution. Lastly, Section 5 concludes the merits of quantile regression in loss reserves model and suggests future development for the model.

\section{Quantile regression}

Most regression models focus on estimating the mean of the data distribution as some functions of predictor variables. Focusing entirely on changes in the mean may, however, fail to identify and distinguish real relationships between variables in heterogeneous distribution. This is particularly problematic for regression models with heterogeneous variances, which are

common in finance and insurance. A regression model with heterogeneous variance implies that there is not a single rate of change that characterizes changes in the data distribution. 
Quantile regression, developed by Koenker and Bassett [13], is an extension of the OLS estimation of the conditional mean to a collection of models with different conditional quantile functions. As the median regression estimator minimizes the symmetrically weighted sum of absolute errors (where the weight equals to 0.5 ) to estimate the conditional median function, other conditional quantile functions are estimated by minimizing an asymmetrically weighted sum of absolute errors, where the weights are functions of the quantile of interest. Suppose we have a model

$$
Y_{i}=x_{i} \beta+\epsilon_{i}
$$

where $\beta$ is an unknown $p \times 1$ vector of regression parameters, $x_{i}$ is a $p \times 1$ vector of predictors, $Y_{i}$ is the outcome variable and $\epsilon_{i}$ is an unknown error term. Ordinary regression minimizes $\sum_{i} \epsilon_{i}^{2}$ whereas median regression minimizes $\sum_{i}\left|\epsilon_{i}\right|$. Koenker and Basset [13] 'tilted' the absolute function called the loss or check or tilt function

$$
\rho_{\tau}\left(\epsilon_{i}\right)=\epsilon_{i}\left(\tau-I\left(\epsilon_{i}<0\right)\right)
$$

to produce the $\tau-t h(\tau \in(0,1))$ conditional quantile of $Y_{i}$ given $x_{i}$

$$
Q \tau\left(y_{i} \mid x_{i}\right)=x_{i} \beta_{\tau}
$$

where $\beta_{\tau}$ minimises

$$
\sum_{i} \rho_{\tau}\left(y_{i}-x_{i} \beta\right)
$$

Note that $0.5 \sum_{i}\left|\epsilon_{i}\right|=\sum_{i} \epsilon_{i}\left(0.5-I\left(\epsilon_{i}<0\right)\right)$ for median regression. The loss function in (1) can be written as

$$
\rho_{\tau}\left(\epsilon_{i}\right)=\epsilon_{i}\left[(\tau-1) I\left(\epsilon_{i}<0\right)+\tau I\left(\epsilon_{i} \geq 0\right)\right],
$$

showing that the weights are symmetric for the median regression $(\tau=0.5)$ and asymmetric otherwise. Their plots are given in Figure 2 for various quantile levels $\tau$ as well as for the OLS regression. 


\section{Loss function}

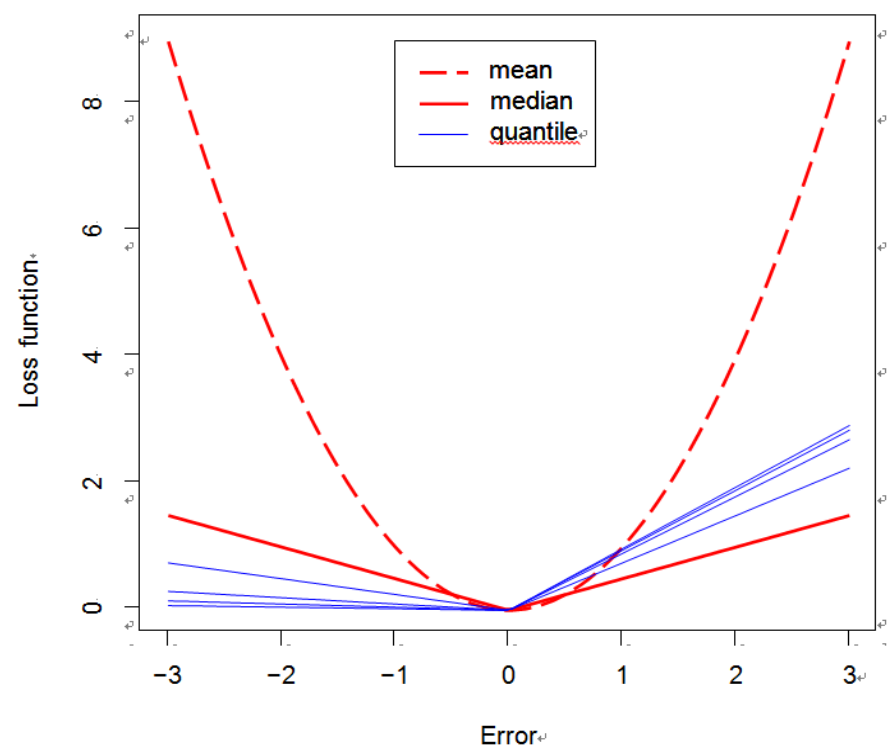

Figure 2: Loss functions for mean, median and quantile $(\tau=0.75,0.9,0.95,0.975)$ regressions.

The minimization of (3) can be performed using the R package quanreg

library(quantreg)

rq(y $\sim x$, tau=taus, method="br")

contrinbuted by Koenker where taus is a vector of quantile levels $\tau$ and "br" is the default method of estimation called the Simplex method which is the modified version of the Barrodale and Roberts algorithm described in Koenker and d'Orey [13], [18]. This method is recommended for moderate sized problems $(n<5,000$ and $p<20$ where $p$ is the number of parameters in the model). It is advantagous to use the Frisch-Newton interior point method "fn" for larger problems and the FrischNewton approach with preprocessing "pfn" (Koenker and Portnoy [16]) for very large problems. Official releases of $\mathrm{R}$ and the install package of quantreg are available at http://lib.stat.cmu.edu/R/CRAN/. See R documentation for other options in quantreg.

In quantile regression, the conditional distribution of $Y$ given $x$ is traced across levels of $\tau$ with $\beta_{\tau}$ estimated in (3) using different values of $\tau$. Hence the model permits parameter heterogeneity across levels of claim as described by the quantile point $\tau$. In the quantile plot of $\hat{\beta}_{\tau}$ against, a significant variation of $\hat{\beta}_{\tau}$ implies that the effect of $x_{i}$ changes as the level of claim increases. Note that all observations are used to estimate the quantile regression parameters and there is no partitioning of data performed on the outcome variable as this would incur sample selection bias. 
Although many papers on quantile regression assume that the errors are independently and identically distributed (i.i.d.), the only necessary assumption concerning $\epsilon_{i}$ is

$$
Q_{\tau}=\left(\epsilon_{i} \mid x_{i}\right)=0
$$

that is, the $\tau$-th conditional quantile of the error term equals to zero. Hence the estimates $\hat{\beta}_{\tau}$ are nonparametric in the sense that no parametric distribution is assumed for $\epsilon_{i}$. The quantile regression estimates in (2) are an ascending sequence of surfaces that are above an increasing proportion of sample observations with increasing quantile levels $\tau$. This operational characteristic extends the concepts of quantiles, order statistics, and rankings to the linear model (Gutenbrunner, Jurecková, Koenker and Portnoy [11]; Koenker and Machado [15]). Quantile regression retains its statistical properties under any linear or nonlinear monotonic transformation of $Y$ as a consequence of this ordering property (Koenker and Machado [15]). Thus it is possible to use a nonlinear transformation, e.g. logarithmic transformation, to estimate linear regression quantiles and then transform back the estimates to the original scale without any loss of information. Moreover parameter estimates $\beta_{\tau}$ have an asymptotic normal distribution

$$
\sqrt{n}\left(\hat{\beta}_{\tau}-\beta_{\tau}\right) \stackrel{d}{\rightarrow} N\left(0, \Sigma_{\tau}\right)
$$

so tests can be constructed using critical values from the normal distribution (Barnes and Hughes [1]).

\section{Expirical Example}

To demonstrate the application of quantile regression in modelling loss reserves, two loss reserves data sets, from Israel and Queensland, Australia respectively, are analyzed. Some general trends are obvious in both data. Given a policy period (year for the Israel data and quarter for the Queensland data), the amount of claims paid follows an increasing trend to a certain lag period and then a decreasing trend thereafter.Table1 reports the means of claim over policy periods for both data and they demonstrate this trend pattern with a peak at the 4th and 9-th lag period respectively. 
Table 1: Average claim across lag year for the loss reserves data from Israel and Queensland, Australia.

\begin{tabular}{cccccc} 
Lag period & Israel & Queensland & Lag period & Israel & Queensland \\
\hline 1 & 2981.28 & 11547661 & 13 & 305.50 & 69890238 \\
2 & 6443.53 & 41908236 & 14 & 259.40 & 63372664 \\
3 & 7631.06 & 58511367 & 15 & 224.00 & 57216920 \\
4 & $* 9243.27$ & 66697013 & 16 & 108.33 & 50841161 \\
5 & 7671.79 & 70051518 & 17 & 38.01 & 44561161 \\
6 & 6229.31 & 73975219 & 18 & 14.00 & 38616361 \\
7 & 5133.75 & 77127866 & 19 & & 34505561 \\
8 & 3268.82 & 77505303 & 20 & & 29871756 \\
9 & 2537.30 & $* 77874046$ & 21 & & 26987458 \\
10 & 1191.22 & 76415123 & 22 & & 20967506 \\
11 & 1058.75 & 76228353 & 23 & & 19152192 \\
12 & 530.43 & 72769273 & 24 & & \\
\hline
\end{tabular}

* Peak of the trend.

On the other hand, there are no obvious trends across policy periods for each lag period. As the level of claim is positive continuous, a logarithm transformation is employed and such transformation will not affect the accuracy of quantile regression. To model the trend pattern of claims across lag-period, we include in the linear function of risk factors the first and second order effects of lag-period and the standardized log initial level of claims or exposure $\mathrm{z}_{i j}$ since the exposure for each policy period affects the levels of claim through out the lag-periods. As a result, the model for the loss reserves data is

$$
Q_{\tau}\left(\ln y_{i j} \mid z_{i j}\right)=\beta_{\tau 0}+\beta_{\tau 1} \times j+\beta_{\tau 2} \times j^{2}+\beta_{\tau 3} \times z_{i j}
$$

where the quantile levels are chosen to include $\tau=0.025,0.05,0.1,0.25,0.75,0.9,0.95,0.99$ apart from the median $\tau=0.5$. This set of quantile levels is adopted in the analyses of both loss reserves data.

For model comparison, three criteria, namely the root mean squared error (RMSE), sum of weighted residuals (SWR) and percentage total (PT), defined as:

$$
\text { RMSE }=\left[\frac{1}{n} \sum_{i=1}^{n} \sum_{j=1}^{n-i+1}\left(y_{i j}-\hat{y}_{i j}\right)^{2}\right]^{\frac{1}{2}},
$$




$$
\begin{gathered}
S W R=\frac{1}{\mathrm{n}} \sum_{i=1}^{n} \sum_{j=1}^{n-i+1} \rho_{\tau}\left(y_{i j}-\hat{y}_{i j}\right), \\
P T=\frac{\sum_{i=1}^{n} \sum_{j=1}^{n-i+1} \hat{y}_{i j}}{\sum_{i=1}^{n} \sum_{j=1}^{n-i+1} y_{i j}} \times 100 \%,
\end{gathered}
$$

are proposed. They measure the model-fit with respect to observations, model-fit with respect to asymmetrically weighted loss function (1) and prediction accuracy by comparing predicted totals with observed totals based on the upper triangle respectively. We note that $S W R$ is only defined for quantile regression models using (1) and model with $R T$ closest to 100 and $R M S E$ and/or $S W R$ the smallest is preferred.

\subsection{Loss reserves data for Israel}

The data are the amount of claims paid to the insureds of an insurance company in Israel during the period of 1978 to 1995 ( $n=18$ years). The upper triangle has $N=171$ observations and the 153 observations in lower triangle are to be estimated. For mathematical convenience, two zero claims are replaced by 0.01 . This data set, as reported in the upper-triangle of Table 2, has been analyzed in Chan, Choy and Makov (2008). There are two extremely large claims, amount to 11,920 and 15,546 dollars, in the 7-th lag year of policy year 1984 and in the 4-th lag year of policy year 1992, respectively. They are outliers as their neighboring claims are much lower in magnitude. These outliers distort the general trend patterns in the data and inflate the standard errors of the model parameters leading to ravaged estimates for loss reserving. These

Table 2: Observed and predicted claims in the run-off triangle using 0.75 quantile level for the Israel

\begin{tabular}{|c|c|c|c|c|c|c|c|c|c|c|c|c|c|c|c|c|c|c|}
\hline & & 2 & & & & & - & 0 & 9 & 0 & $\cdots$ & & & & 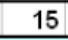 & 16 & & \\
\hline 78 & 23 & 32 & 72 & 72 & 7631 & 3855 & 3252 & 3 & 8 & & & & & & 05 & & 76 & \\
\hline & 85 & & & & & & & & & & & & & & & 1 & & \\
\hline 80 & 77 & 89 & 46 & & & & & & & 6 & & & & 10 & 10 & 31 & & \\
\hline 81 & 88 & 8089 & 339 & & 7560 & 383 & 18 & & 575 & 1985 & & & 38 & 45 & 115 & & & \\
\hline 82 & 94 & 369 & & & & 419 & & & & & & & & 359 & the & & & \\
\hline 1 & 00 & & & & & & & & & & & & & 39 & & & & \\
\hline 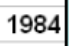 & 42 & & & & & & & & & & & & & & & & & \\
\hline 85 & 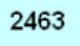 & & & & & & & & & & & & & & & & & \\
\hline 86 & 67 & & & & & & & & & 903 & & & & & & & & \\
\hline 887 & 09 & & & 6 & 647 & 757 & 5 & 51 & 71 & 1 & & & & & & & & \\
\hline 988 & (60, & & & & & & 62 & & & & & & & & & & & \\
\hline 89 & 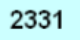 & & & & & 92 & & $\sqrt{52}$ & & & & & & & & & & \\
\hline 90 & & & & & & 100 & & & & & & & & & & & & \\
\hline & & & & & & & & & & & & & & & & & & \\
\hline & 95 & & & & & & & & & & & & & & & & & \\
\hline 1000 & 155 & & & & & & & & & & & & & & 8 & & & 14 \\
\hline 1994 & 626 & & & & & 76 & & 52 & 35 & & & & & & & 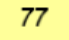 & & 14 \\
\hline 95 & 27 & 98 & & & 451 & 7723 & 6599 & 5273 & 3939 & 2751 & 1796 & 1097 & 626 & 334 & 167 & 78 & 34 & 74 \\
\hline
\end{tabular}
loss reserves data. 
two outliers can be seen in Figure 3 which plots the trend of claims and their means in Table 1 across lag-year. For robustness consideration, Chan, Choy and Makov [7] suggested using the GT distribution which includes both platykurtic and leptokurtic distributions to accommodate these irregular claims.

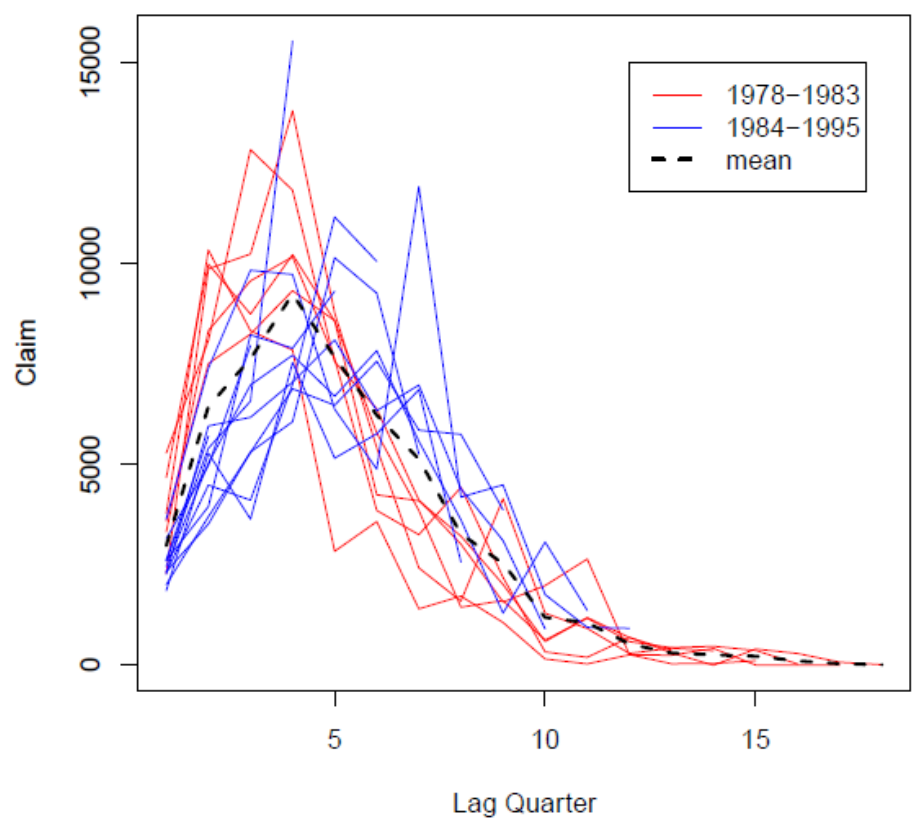

Figure 3: Claim across lag period for the loss reserves data from Israel.

Figure 3 further shows that the claim payments for each policy year follows two distinct increasing-then-decreasing trend patterns: during 1978 to 1983, the trend increases to a high peak at approximately the 4-th lag year and then decreases thereafter whereas during 1984 to 1995, the trend increases slowly to a lower peak at about the 6-th lag year and then decreases. Hence Chan, Choy and Makov [7] further proposed a threshold model to incorporate a model shift after 1983 and a state space model to account for the interaction between the policy-year and lag-year effects. The proposed threshold state space model with GT errors (called GT model) was implemented using Bayesian approach. They demonstrated that the GT model out-performed the popular chain-ladder (CL) model in model-fit for claims in the upper run-off triangle. Refer to Section 6.4 of Chan, Choy and Makov [7] for details of the CL model. Although the data are not adjusted for inflation, it successfully demonstrates the ability of GT model to capture various sources of variability. We propose modelling the data using quantile regression. Resultant regression quantiles are graphed in Figure 4 (a) and (b) for the log claim and claim respectively. 
(a) Quantile regression of Log Claim at $z_{-} i j=0$ for Israel

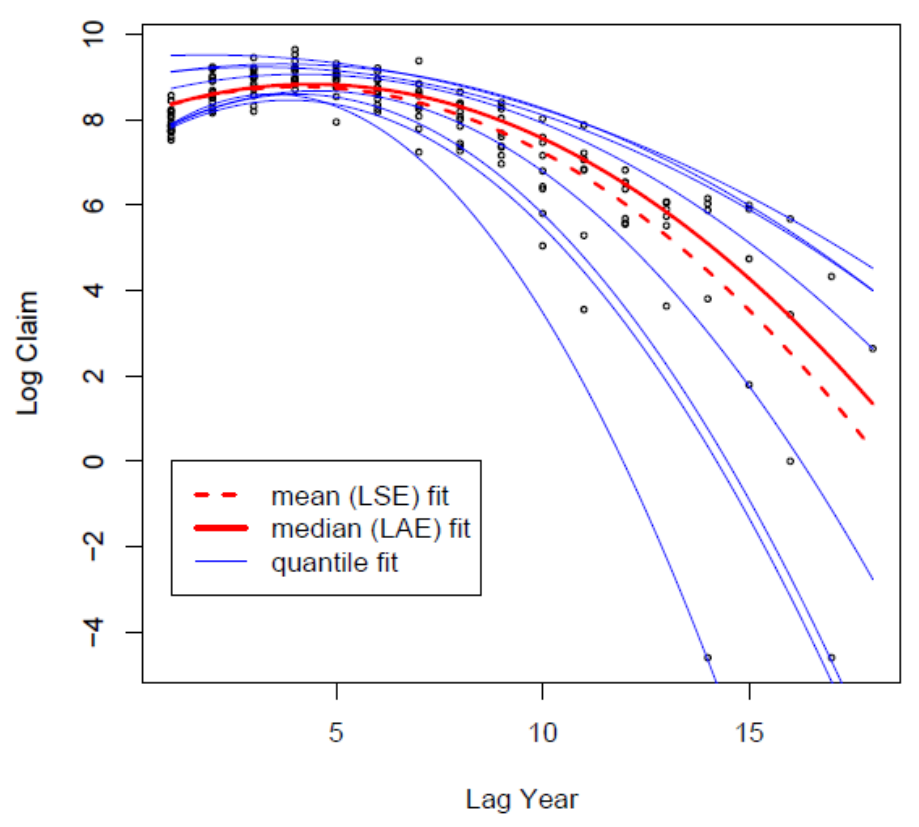

(b) Quantile regression of Claim at $z_{-} i j=0$ for Israel

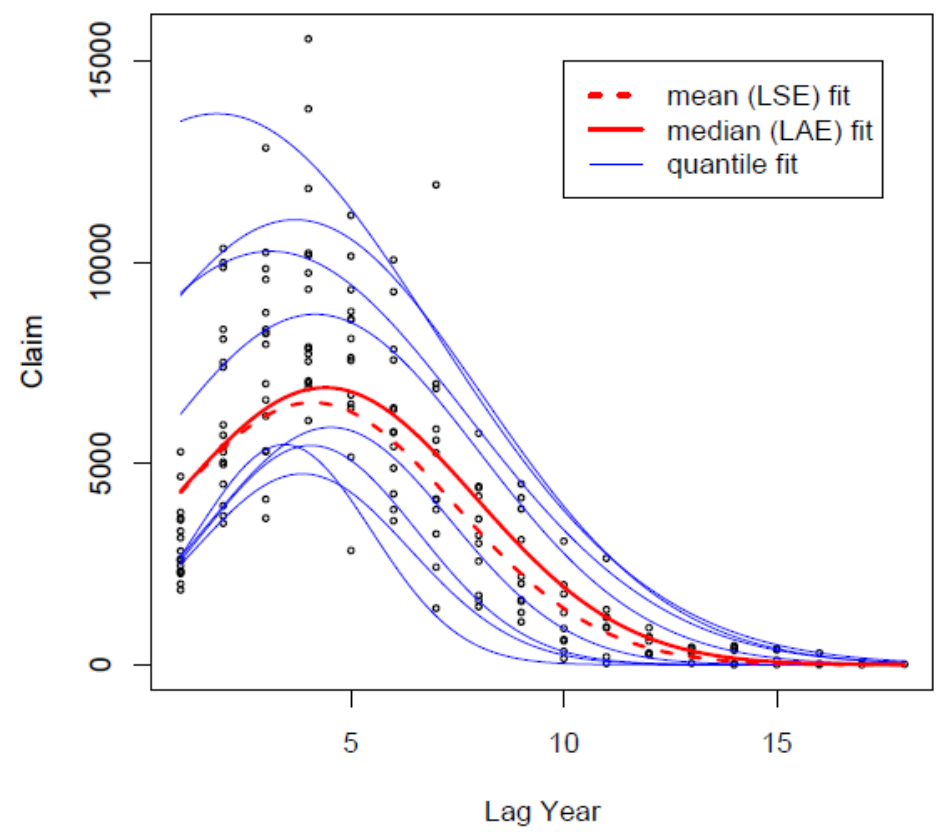

Figure 4: Quantile regression lines for (a) the logarithm of claims, $\ln Y_{i j}$, and (b) claims, $Y_{i j}$, for Israel loss reserves data. 
The quantiles for log claim show less variation in higher level claims and more variation in lower level claims showing a phenomenon of concern when logarithmic transformation is taken. The asymmetric variance violates the constant variance assumption in the mean regression model, in particular the GT model, but such assumption is not required in quantile regression, an advantage of employing quantile regression over mean regression for modelling loss reserves data. Moreover the two zero outliers shift the error distribution to negatively skewed which violates the GT error assumption in the GT model. On the other hand, they affect only the lower quantiles in quantile regression and such effect disappears after taking exponential transformation, demonstrating another advantage of using quantile regression. Some quantiles cross over in Figure 4(a) and the crossover effect becomes more apparent in Figure 4(b) after taking exponential transformation. Now the quantiles for larger claims show more variation and such variation gradually disappears across lag-year when the level of claims drops to zero. Moreover the smaller gaps between lower quantiles and wider gaps between higher quantiles show that the conditional distribution of claims is heavily skewed to the right, that is, the risk of expensive losses is likely to be higher during the early lag-years. To maintain solvency and prevent the risk of bankruptcy for a company, perhaps insurers should achieve a higher level of risk protection by reserving fund at the quantile level $\tau=0.75$ instead of at the mean in Chan,Choy and Makov [7].

\subsection{Loss reserves data for Queensland, Australia}

The data are the amount of total incurred cost for the compulsory third party (CTP) policies in Queensland, Australia. Observed figures are defined as case estimates plus payment to date for each claim. All values have been inflated to December 2008 dollars. The data is summarized by policy quarters (instead of year) and development/lag quarters in the upper triangle of Table 3. Since there is one major legislative change in December 2002, the data start from 2002 onward to avoid the influence of legislative change. Covering the period of December 2002 to June 2008, the data contain 23 quarters and 276 observations. The aim of the analysis is to predict the 253 future claims in the lower run-off triangle.

The plot of aggregated claims across lag-quarter for each policy quarter is shown in Figure 5. The plot shows that during the first period of Dec 2002 to Jun 2003, trend rises up very fast to a high peak at about the 4-th lag quarter and levels off till the 12-th lag quarter before it drops, during the second period of Sept 2003 to Sept 2005, the trend shows a more gentle increase to a lower peak at approximately the 10-th lag quarter and then a decrease whereas during the last period of Dec 2005 to Jun 2008, the trend rises up faster again till the 7-th lag quarter and then declines thereafter. There is no obvious outliers in the data to distort the trend patterns. Regression quantiles are plotted in Figures 6(a) and (b) for the log claim and claim respectively. 
Figure 5: Claim across lag period for the loss reserves data from Queensland, Australia.

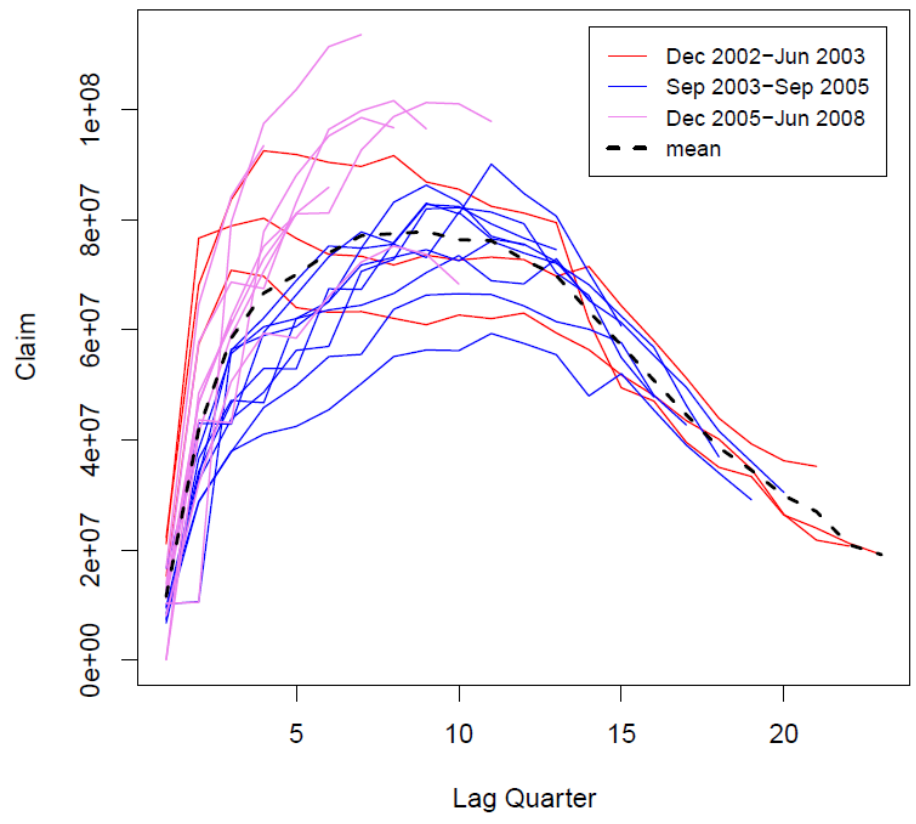




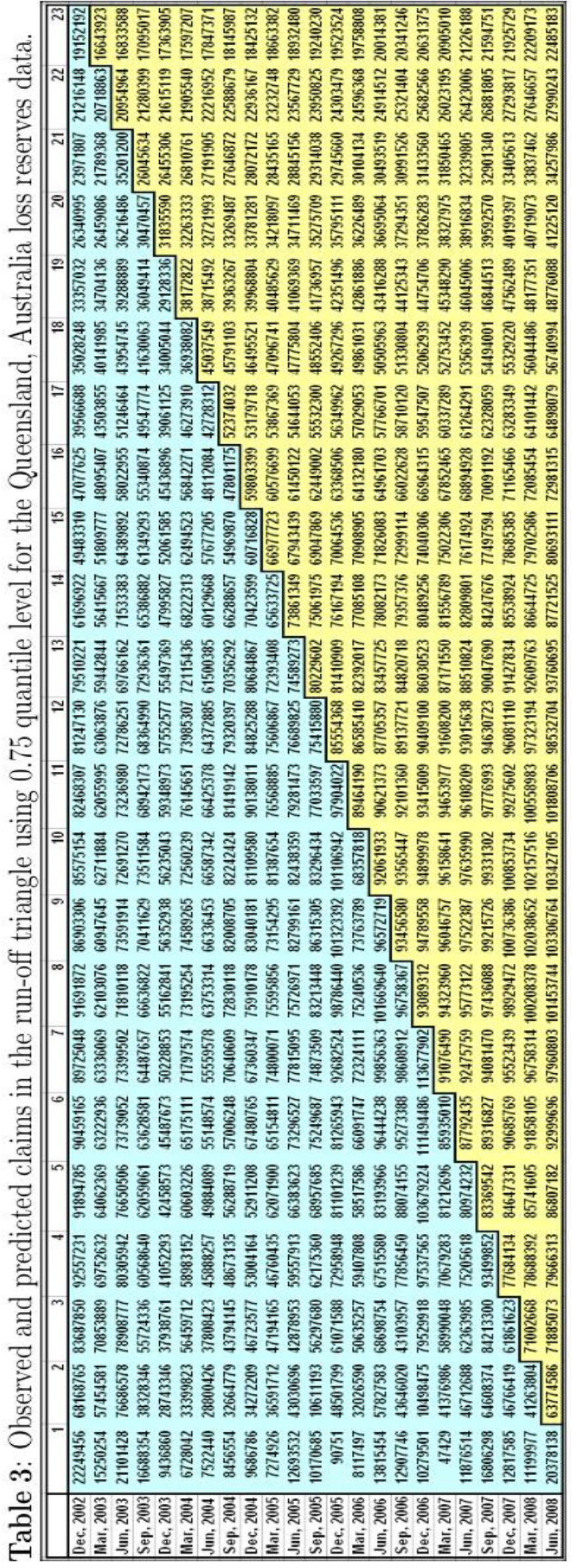


(a) Quantile regression of Log Claim at $\mathrm{z}_{-} \mathrm{ij}=0$ for Queensland, Australia

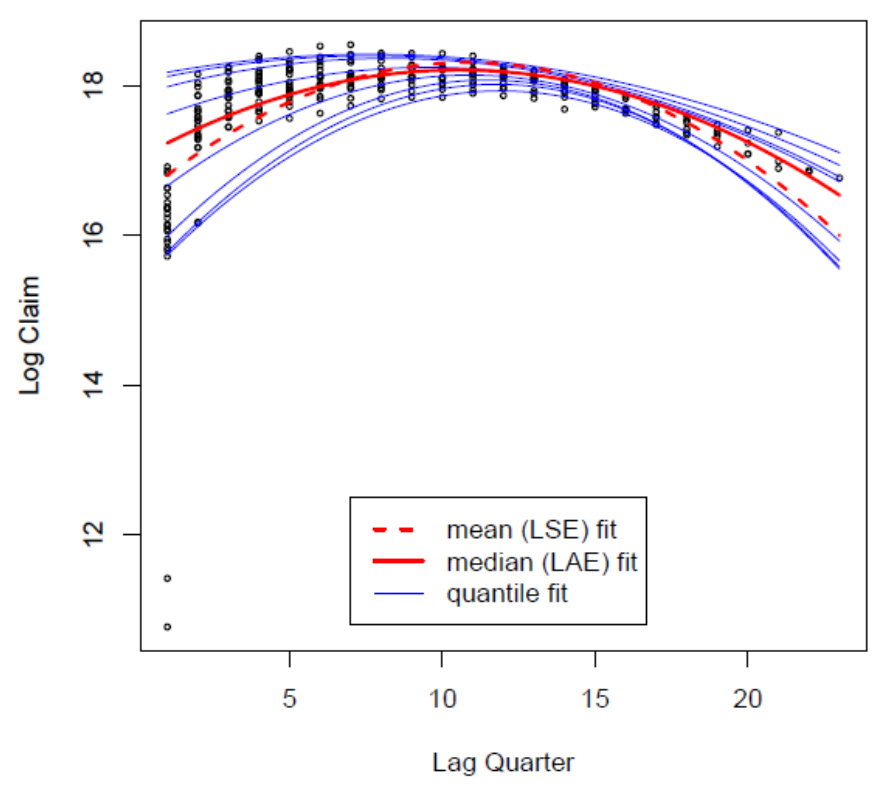

(b) Quantile regression of Claim at $z_{-} i j=0$ for Queensland, Australia

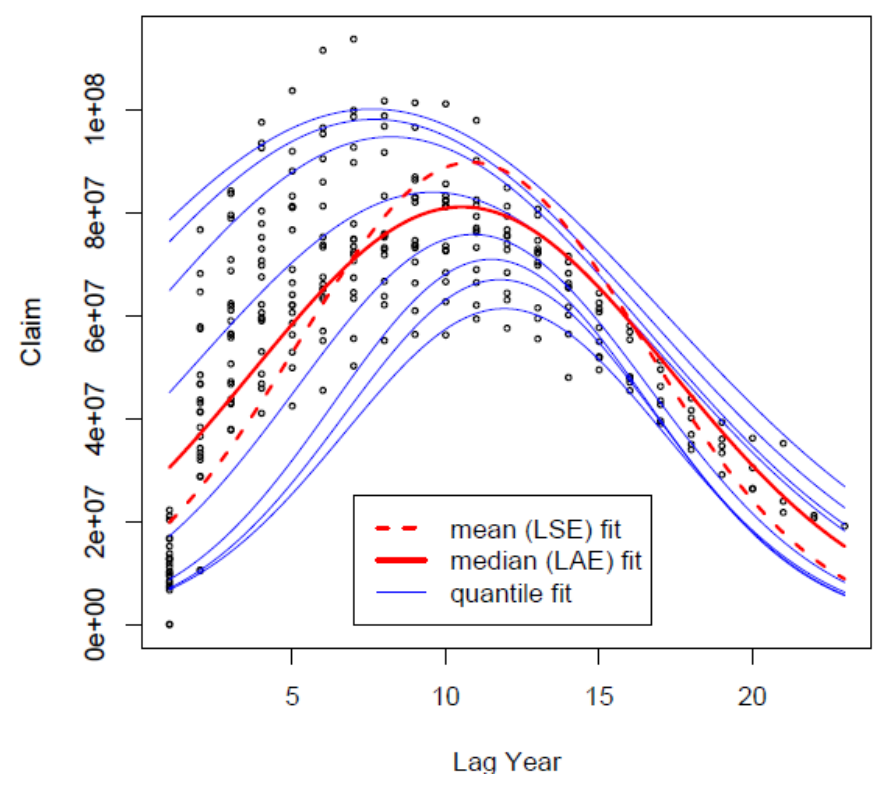

Figure 6: Quantile regression lines for (a) the logarithm of claims, $\ln Y_{i j}$, and (b) claims, $Y_{i j}$, for Queensland, Australia loss reserves data 
After taking logarithm transformation of the data, heterogeneous variance is again observed in Figure 6(a), particularly due to the two extremely low outliers in the 1st lag quarter. While they deflate the mean more, they affect only the lower quantiles. After transforming back, the two outliers are no longer extreme while all other lag-one observations are closely located in the lower quantiles. There is no crossover in both Figures 6(a) and (b) but the trend of mean is very different from that of median: it rises from a lower level at a faster rate to reach a higher peak and then decreases at a faster rate. These two distinct trend patterns, giving very different claim predictions, are caused by the two extreme low outliers in the first lag quarter, high outliers around the 6-th to 11-th lag quarters and low outliers again around the 12-th to 14-th lag quarters, leading to steeper trends than the median which are more robust to outliers. Regression quantiles are now spacing more even on the two sides of the median so that the conditional loss distribution is about symmetric. Forecast using quantile level $\tau=0.75$ is described in the next section.

\section{Forecast}

The aim of the analyses is to forecast future claims in the lower triangle of the loss reserves data using (4) and the $75 \%$ regression quantile. The parameter estimates are given in Tables 4 and 5 for the two loss reserves data. Forecasts of loss reserves are given in the lower triangle of Tables 2 and 3.

Entries in the first diagonal of the lower triangle (highlighted in dark yellow in Table 2 for illustration) are the one-period ahead forecasts over all policy periods and its total is the amount of reserves insurers to pay for the claims in one period time. Similarly, the second diagonal total gives the reserves for the second period in the future using the two-period ahead forecast and hence the sum of all diagonal totals or all entries in the lower triangle gives the total reserves for the future $(n-1)$ periods using the $(n-1)$-period ahead forecast. Tables 6 and 7 report the diagonal totals and their sum across levels of upper quantiles as well as those using the mean and median regressions for the two data sets. 
Table 4: Parameter estimates and their s.e. (in italic) for the Israel loss reserve data.

\begin{tabular}{ccccccccccc}
\hline$\tau$ & mean & 0.025 & 0.05 & 0.1 & 0.25 & 0.5 & 0.75 & 0.9 & 0.95 & 0.975 \\
\hline$\beta_{0}$ & 8.0451 & 7.2016 & 7.2897 & 7.2969 & 7.3876 & 8.0538 & 8.4902 & 9.0085 & 8.9590 & 9.4586 \\
& 0.2532 & 0.3241 & 0.1567 & 0.0890 & 0.1343 & 0.2110 & 0.1934 & 0.1600 & 0.0507 & 0.1428 \\
$\beta_{1}$ & 0.3602 & 0.8176 & 0.6090 & 0.6439 & 0.5717 & 0.3562 & 0.2796 & 0.1469 & 0.1909 & 0.0711 \\
& 0.0748 & 0.1841 & 0.1133 & 0.0716 & 0.0714 & 0.0756 & 0.0627 & 0.0449 & 0.0432 & 0.0536 \\
$\beta_{2}$ & -0.0440 & -0.1189 & -0.0789 & -0.0793 & -0.0631 & -0.0405 & -0.0336 & -0.0236 & -0.0259 & -0.0192 \\
& 0.0046 & 0.0205 & 0.0179 & 0.0111 & 0.0074 & 0.0062 & 0.0047 & 0.0029 & 0.0030 & 0.0033 \\
$\beta_{3}$ & 0.0039 & 0.2296 & 0.1234 & 0.1245 & 0.0419 & 0.0162 & 0.0197 & 0.0109 & 0.0404 & -0.0224 \\
& 0.0939 & 0.1338 & 0.0195 & 0.0268 & 0.0471 & 0.0425 & 0.0467 & 0.0403 & 0.0509 & 0.0710 \\
\hline \multirow{2}{*}{$x_{p}$} & 4.091 & 3.438 & 3.859 & 4.058 & 4.530 & 4.399 & 4.160 & 3.111 & 3.685 & 1.853 \\
$y_{p}$ & 6516 & 5471 & 4744 & 5449 & 5899 & 6886 & 8707 & 10271 & 11056 & 13690 \\
\hline$R M S E$ & 2101 & 3096 & 3077 & 2737 & 2429 & $2027 \dagger$ & 2273 & 3311 & 3679 & 5397 \\
$S W R$ & - & 0.067 & 0.110 & 0.167 & 0.271 & 0.294 & 0.205 & 0.100 & 0.056 & $0.030 \dagger$ \\
$P T$ & 86.73 & 50.68 & 51.51 & 59.28 & 70.36 & $94.42 \dagger$ & 124.64 & 151.95 & 164.89 & 193.74 \\
\hline
\end{tabular}

$\dagger$ Best across quantile level $\tau$.

Table 5: Parameter estimates and their s.e. (in italic) for the Queensland, Australia loss reserve data.

\begin{tabular}{ccccccccccc}
\hline$\tau$ & mean & 0.025 & 0.05 & 0.1 & 0.25 & 0.5 & 0.75 & 0.9 & 0.95 & 0.975 \\
\hline$\beta_{0}$ & 16.482 & 15.314 & 15.340 & 15.572 & 16.348 & 17.018 & 17.470 & 17.876 & 18.035 & 18.101 \\
& 0.1066 & 1.9657 & 1.6000 & 0.1704 & 0.2044 & 0.0806 & 0.0858 & 0.1050 & 0.0904 & 0.0786 \\
$\beta_{1}$ & 0.3380 & 0.4399 & 0.4555 & 0.4367 & 0.3297 & 0.2266 & 0.1621 & 0.1193 & 0.0955 & 0.0844 \\
& 0.0245 & 0.3445 & 0.2765 & 0.0379 & 0.0351 & 0.0756 & 0.0135 & 0.0181 & 0.0181 & 0.0155 \\
$\beta_{2}$ & -0.0156 & -0.0185 & -0.0194 & -0.0190 & -0.0151 & -0.0108 & -0.0085 & -0.0073 & -0.0062 & -0.0056 \\
& 0.0012 & 0.0150 & 0.0118 & 0.0018 & 0.0015 & 0.0062 & 0.0005 & 0.0008 & 0.0009 & 0.0007 \\
$\beta_{3}$ & 0.0231 & 0.0302 & 0.0792 & 0.0972 & 0.0635 & 0.1180 & 0.0795 & 0.0377 & 0.0501 & 0.0483 \\
& 0.0429 & 0.2876 & 0.1764 & 0.0404 & 0.0294 & 0.0425 & 0.0244 & 0.0203 & 0.0214 & 0.0206 \\
\hline \multirow{2}{*}{$x_{p}$} & 10.83 & 11.91 & 11.77 & 11.47 & 10.89 & 10.53 & 9.57 & 8.23 & 7.66 & 7.61 \\
$y_{p}^{*}$ & 89.76 & 61.41 & 67.04 & 70.92 & 75.73 & 81.13 & 83.94 & 94.73 & 98.07 & 100.13 \\
\hline$R M S E^{*}$ & 17.84 & 31.84 & 29.56 & 26.53 & 19.41 & $14.18 \dagger$ & 16.18 & 25.55 & 30.68 & 33.25 \\
$S W R$ & - & 0.050 & 0.064 & 0.089 & 0.134 & 0.133 & 0.094 & 0.047 & 0.025 & $0.013 \dagger$ \\
$P T$ & 95.69 & 57.23 & 61.53 & 66.96 & 81.26 & $98.89 \dagger$ & 111.78 & 132.08 & 140.53 & 145.65 \\
\hline
\end{tabular}

$*$ RMSE $=R M S E^{*} \times 10^{6}$ and $y_{p}=y_{p}^{*} \times 10^{6} . \quad \dagger$ Best across quantile level $\tau$. 
144 Predicting loss reserves using quantile regression Running title: Quantile regression loss reserve models

Table 6: Estimates of loss reserve at diagonals of lower triangle and their total for the Israel loss reserve data

\begin{tabular}{crrrrrr}
\hline Diag. & mean & 0.5 & 0.75 & 0.9 & 0.95 & 0.975 \\
\hline 1 & 42827.36 & 47791.33 & 62810.29 & 74938.37 & 82037.63 & 93548.19 \\
2 & 37473.91 & 42429.24 & 55506.57 & 65046.44 & 72102.71 & 79647.80 \\
3 & 31310.20 & 36155.34 & 47318.70 & 54858.87 & 61510.45 & 66098.86 \\
4 & 24813.25 & 29391.11 & 38739.55 & 44850.59 & 50786.19 & 53390.20 \\
5 & 18543.33 & 22670.38 & 30339.01 & 35473.23 & 40481.64 & 41915.07 \\
6 & 13003.17 & 16515.61 & 22650.78 & 27091.38 & 31077.77 & 31948.64 \\
7 & 8520.76 & 11319.75 & 16074.50 & 19946.16 & 22934.49 & 23615.60 \\
8 & 5199.97 & 7275.78 & 10816.59 & 14136.90 & 16242.43 & 16908.55 \\
9 & 2947.18 & 4373.71 & 6886.69 & 9631.61 & 11020.65 & 11714.45 \\
10 & 1547.73 & 2453.39 & 4140.85 & 6299.18 & 7153.57 & 7842.28 \\
11 & 751.66 & 1281.65 & 2347.20 & 3948.03 & 4434.53 & 5064.42 \\
12 & 336.98 & 622.32 & 1251.70 & 2365.53 & 2618.63 & 3147.53 \\
13 & 139.17 & 280.21 & 626.21 & 1349.75 & 1467.92 & 1873.74 \\
14 & 52.77 & 116.49 & 292.27 & 727.72 & 775.19 & 1059.57 \\
15 & 18.21 & 44.25 & 125.60 & 364.47 & 379.53 & 558.42 \\
16 & 5.55 & 14.84 & 47.75 & 161.94 & 164.30 & 262.26 \\
17 & 1.30 & 3.82 & 13.85 & 54.52 & 54.12 & 92.74 \\
\hline Total & 187492.50 & 222739.20 & 299988.12 & 361244.70 & 405241.77 & 438688.30 \\
\hline & & & & & &
\end{tabular}


Table 7: Estimates of loss reserve at diagonals of lower triangle and their total for the Queensland, Australia loss reserve data.

\begin{tabular}{crrrrrr}
\hline Diag. & mean & 0.5 & 0.75 & 0.9 & 0.95 & 0.975 \\
\hline 1 & 1222212592 & 1374445643 & 1440262827 & 1559190250 & 1669416383 & 1740823245 \\
2 & 1198952810 & 1352347499 & 1396411092 & 1490290616 & 1592072003 & 1659684648 \\
3 & 1167171458 & 1320046559 & 1343727980 & 1414052606 & 1507904148 & 1572249246 \\
4 & 1125788993 & 1276962117 & 1282355378 & 1331148747 & 1417691635 & 1479266551 \\
5 & 1074121052 & 1222995146 & 1212818621 & 1242527192 & 1322454610 & 1381701174 \\
6 & 1012032540 & 1158553924 & 1135997495 & 1149367638 & 1223399727 & 1280678931 \\
7 & 940047344 & 1084484697 & 1053022477 & 1052996269 & 1121812541 & 1177374833 \\
8 & 859406748 & 1002092012 & 965259125 & 954846774 & 1019032384 & 1072998848 \\
9 & 772047636 & 913182549 & 874329539 & 856441878 & 916469564 & 968833421 \\
10 & 680471240 & 819907669 & 781984852 & 759302188 & 815513917 & 866146420 \\
11 & 587529960 & 724542424 & 689942391 & 664843291 & 717423811 & 766079635 \\
12 & 496179230 & 629417178 & 599862208 & 574349572 & 623332649 & 669670593 \\
13 & 409211893 & 536834738 & 513320993 & 488952690 & 534257211 & 577874405 \\
14 & 329006453 & 448876404 & 431698241 & 409572794 & 451036828 & 491502098 \\
15 & 257334931 & 367194827 & 356047600 & 336858957 & 374257407 & 411136176 \\
16 & 195268661 & 292972645 & 287091999 & 271195317 & 304266529 & 337146485 \\
17 & 143186195 & 227055909 & 225347099 & 212772548 & 241282519 & 269811822 \\
18 & 100831436 & 169803067 & 171008766 & 161547853 & 185314824 & 209221255 \\
19 & 67443589 & 121089562 & 123951150 & 117260175 & 136162386 & 155261634 \\
20 & 41927685 & 80457649 & 83830372 & 79495841 & 93488102 & 107691663 \\
21 & 23025954 & 47285608 & 50199416 & 47758190 & 56899103 & 66223418 \\
22 & 9452673 & 20778153 & 22485183 & 21471520 & 25921411 & 30482882 \\
\hline Total & 12712651072 & 15191325979 & 15040954802 & 15196242905 & 16349409692 & 17291859386 \\
\hline & & & & & &
\end{tabular}

\subsection{Loss reserves data for Israel}

The parameter estimates as reported in Table 4 and their confidence intervals (CIs) across quantile levels $\tau$ are graphed in Figure 7 . 
0

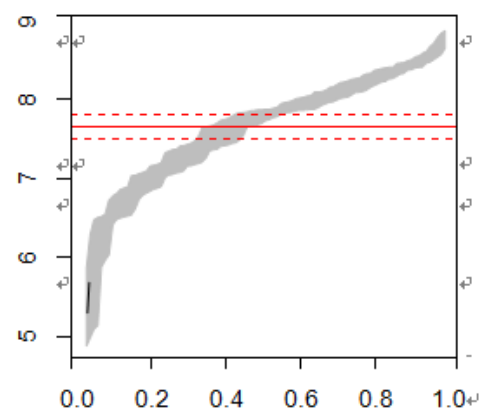

2

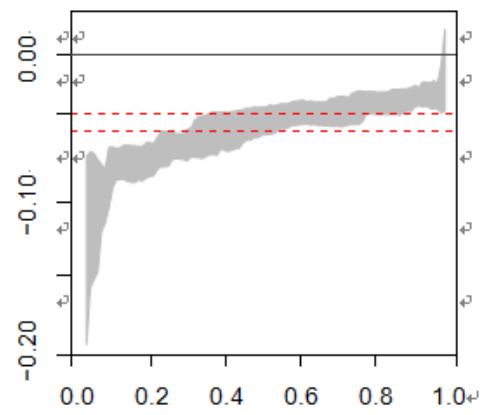

1.

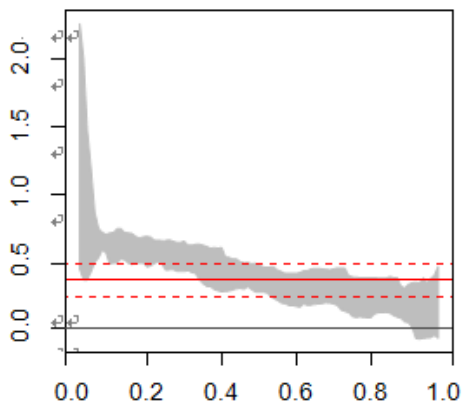

3.

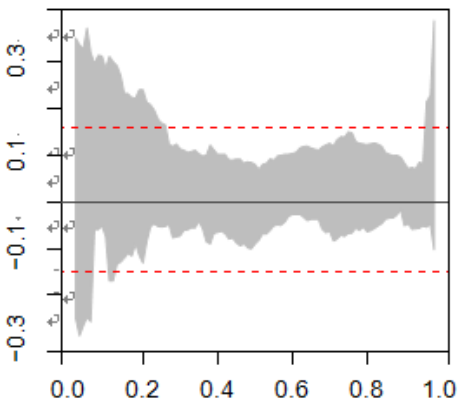

Figure 7: Parameter estimates and their $95 \%$ confidence intervals across quantiles for Israel loss reserves data.

The CIs for $\beta_{0}, \beta_{1}$ and $\beta_{2}$ are very sharp showing high levels of significance except for the very low quantiles and they change in sign and magnitude across quantile levels $\tau$. Koenker [18] remarked that the endpoints of the CIs are not always symmetric about the estimate because of the skewed sampling distribution of the estimates especially for smaller sample and more extreme quantiles. In this case, the sampling variation for the quantiles can change rapidly over a short interval of quantiles. As $\hat{\beta}_{1}$ and $\hat{\beta}_{2}$ describe the trend of claims across lag-years, their distinct estimates on different quantile levels trace a gradual change in trend pattern from a higher peak $\left(y_{p}\right)$ at earlier lag-year $\left(x_{p}\right)$ to a lower peak at later lag-year as the quantile level decreases. The coordinates of the peak $\left(x_{p}, y_{p}\right)$ are reported in Table 4 . This result is supported by the data plot in Figure 3, agrees with the result of the sophisticated GT model but is achieved by a single quantile regression model. Lastly $\beta_{3}$ which measures the effects of initial claim levels or exposure on claim sizes, has positive but insignificant effects over nearly all quantile levels. Despite insignificant, it shows intuitively how the later levels of claim depend on the initial claim size just after policies were made.

The model performance measures RMSE, SWR and $P T$ are reported in Table 2. The corresponding RMSE and $P T$ values for model using GT distribution are $(1258.7,97.62)$ and for model using CL method are $(1976.9,97.71)$ respectively. Being the most sophisticated model, 
the GT model provides the best model-fit according to RMSE. Both the GT and CL models perform the best in terms of $P T$ whereas the median regression model is preferred among all quantile regression models. However all the three models give underestimation of total claims in the upper triangle. We note that the $75 \%$ quantile regression model performs slightly less satisfactory which can be explained in Figure 8 by the mild overestimates for low claims and underestimates for high claims.

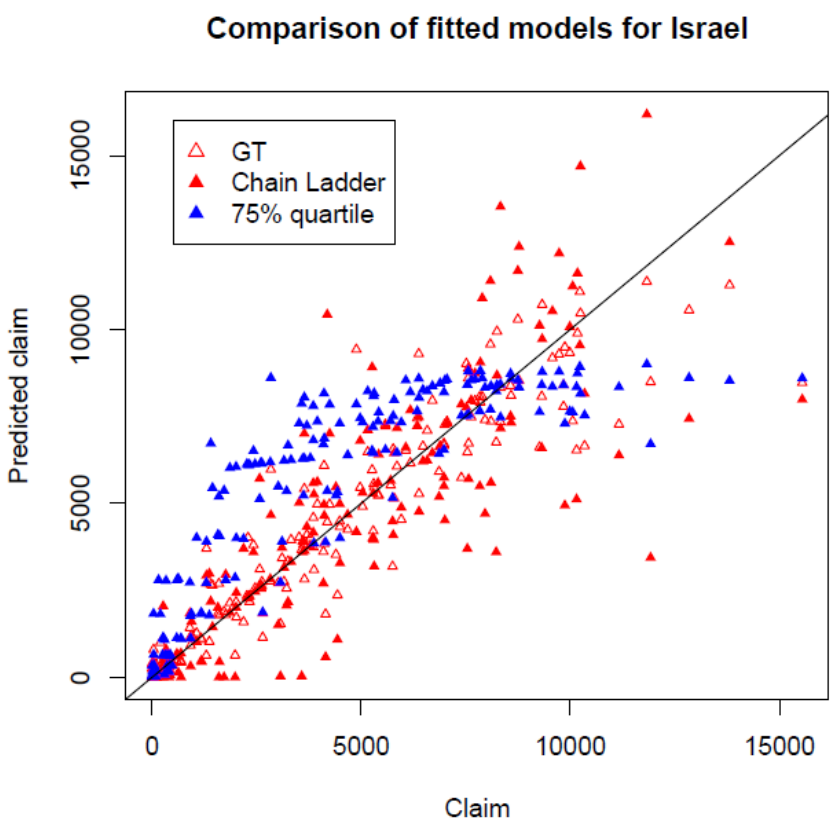

Figure 8: Predicted claim again observed claim in the upper triangle for Israel loss reserves data.

While over- and underestimations are expected using higher quantile levels, the $75 \%$ quantile regression model gives a slight overestimate of overall total in the upper triangle as compared to the GT, CL and median regression models which give under- estimates. The slight overestimate is perhaps a realistic level of loss reserve fund for insurers to maintain solvency. Lastly the $97.5 \%$ quantile regression model provides the most minimization of the asymmetric loss function (1). Figure 9 plots the residuals of quantile regression model across quantile level $\tau$. It can be seen that the distribution changes from right-skewed to left-skewed on increasing $\tau$. 

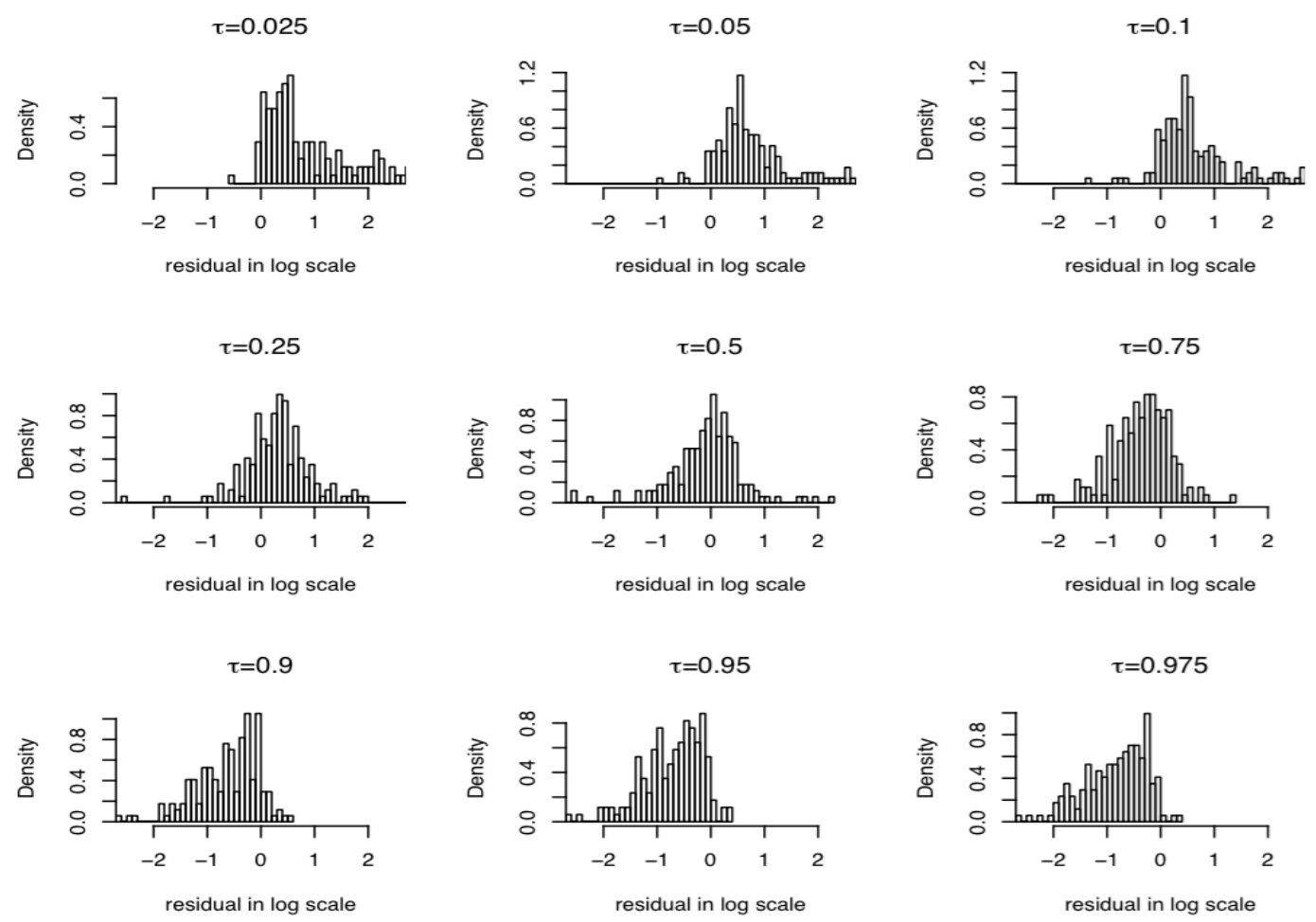

Figure 9: Residuals in the upper triangle of quantile regression models across quantile levels $\tau$ for Israel loss reserves data.

Predicted $i$-year ahead claim totals $(i=1, \ldots, n-1)$ using the mean, median and (upper) quantile regressions and their overall totals across quantile levels are reported in Table 6 and graphed in Figure 10(a). 
Prediction of diagonal totals in the lower triangle

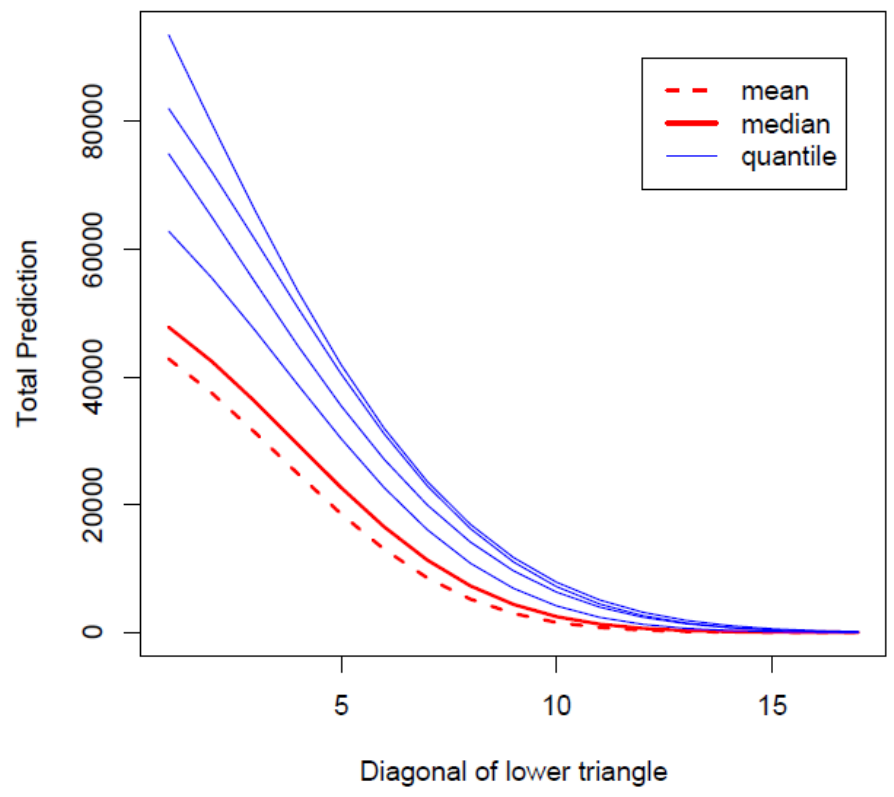

(b) Prediction of diagonal totals in the lower triangle for Queensland, Australia

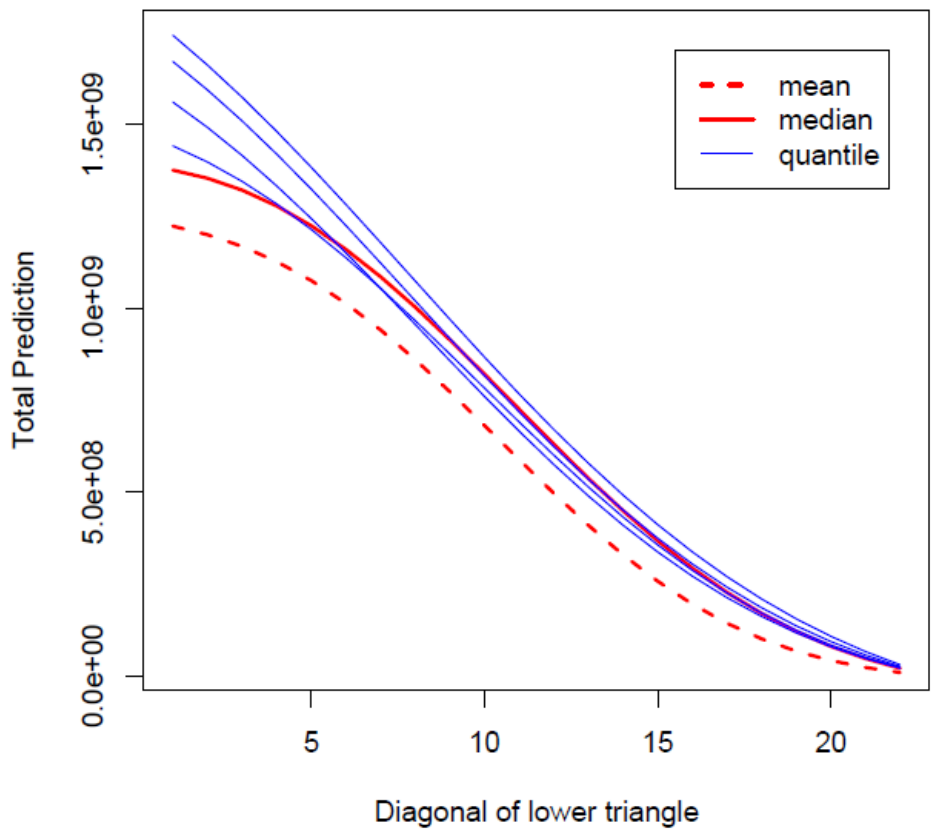

Figure 10: Prediction of diagonal totals in the lower triangle for (a) Israel and (b) Queens- land, Australia loss reserves data. 
The level of reserves increases with increasing quantile level $\tau$ and decreasing $i$-th lag year diagonal in the lower triangle, but the gaps between the mean, median and successive pairs of quantiles are substantial showing that prediction using the mean may underestimate the level of loss reserves resulting in sufficient fund reserved for future claims. Chan, Choy and Makov [7] predicted the total outstanding claims in the lower triangle to be 296,159 dollars with a standard error of 123,867 dollars. Our projected totals using a simple mean regression and $75 \%$ quantile regression are 187,493 dollars and 299,988 dollars respectively, with the latter being similar to the projected total using the GT model.

\subsection{Loss reserves data for Queensland}

Again, the parameter estimates are reported in Table 5 and their CIs across quantile levels $\tau$ are graphed in Figure 11.
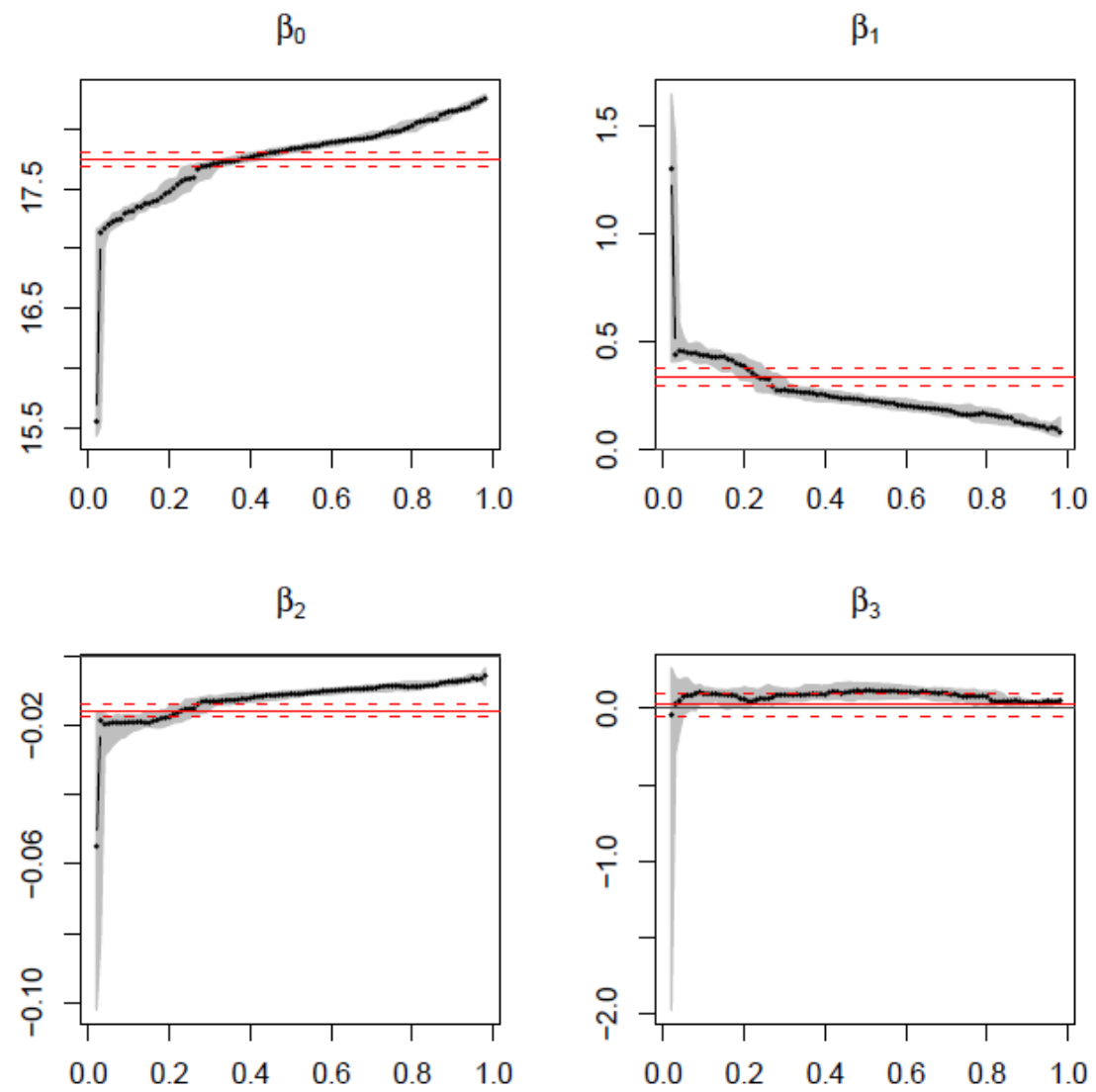

Figure 11: Parameter estimates and their 95\% confidence intervals across quantiles for Queensland loss reserves data. 
Trends of CIs across $\tau$ are similar to those using Israel loss reserves data but the CIs are more sharp except for very low quantiles. Now the exposure effect is more significant, indicating that higher level of exposure is associated with larger claim throughout the lag-quarters. Trends of regression quantiles in Figure 6(b) again follow the pattern that higher peak occurs at earlier lagquarter and lower peak at latter lag-quarter as the quantile level decreases. Table 5 shows that the median regression and $75 \%$ quantile regression are the first and second best models according to $R M S E$. While the former model gives an underestimate of total claim in the upper triangle according to $P T$ but the latter model gives an overestimate of only $11 \%$ above the actual total, the latter model is chosen to forecast future claims for solvency consideration. The model suggests that 15,040,954,802 dollars should be saved for the future 22 quarters (5.5 years). Predicted $i$-period ahead claim totals $(i=1, \ldots, n-1)$ using the mean, median and (upper) quantile regression and their overall totals across quantile levels are reported in Table 7 and graphed in Figure 10(b). The median and quantile lines show similar decreasing trends as in Figure 10(a) for Israel data. However the mean regression line crosses over some quantiles showing that the predicted diagonal totals using the mean regression will not be seriously underestimated. Again, SWR shows that the $97.5 \%$ quantile regression model provides the most minimization of (1) and Figure 12 which plots the residual distributions among different quantile regression models shows the change of shape from right-skewed to left-skewed on increasing quantile level $\tau$. 


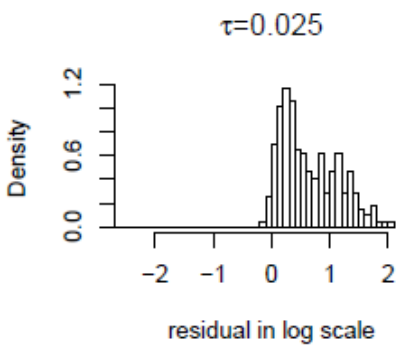

$\tau=0.25$

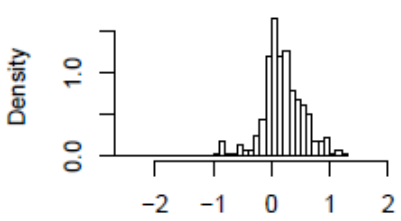

residual in log scale

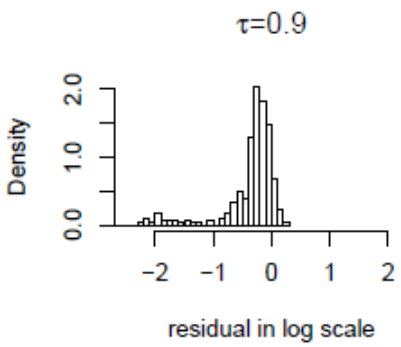

$\tau=0.05$

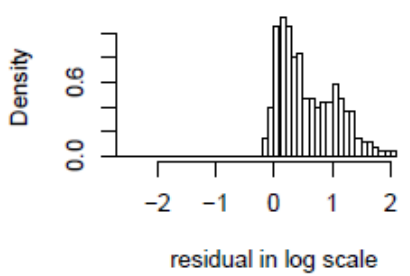

$\tau=0.5$

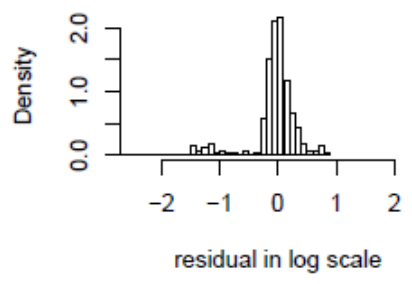

$\tau=0.95$

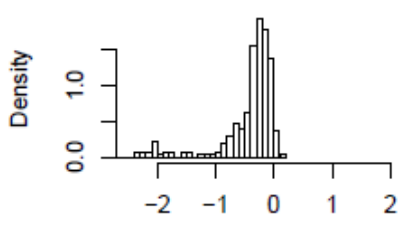

residual in $\log$ scale

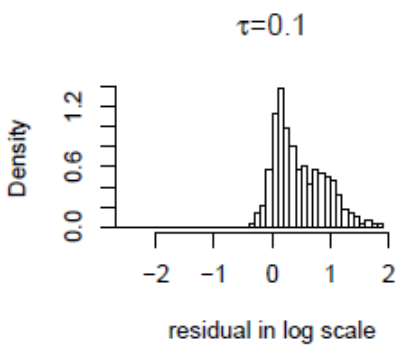

$\tau=0.75$

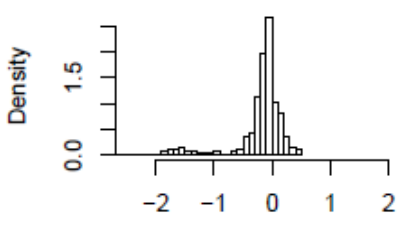

residual in $\log$ scale

$\tau=0.975$

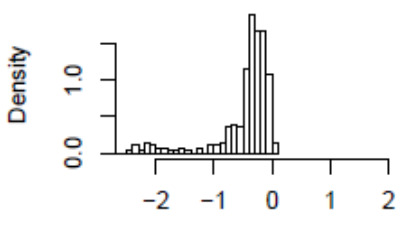

residual in log scale

Figure 12: Residuals in the upper triangle of quantile regression models across quantile levels $\tau$ for Queensland loss reserves data.

\section{Conclusion}

As insurers receive premiums from policyholders in advance to pay for the future claims on losses specified in insurance contracts in return, they must have the necessary loss reserves to pay for these outstanding claims and settlement costs incurred. To provide sufficient reserves for outstanding claims, prediction of over-claimed is more important and hence the focus of loss reserves model lies more on the upper tails of the conditional distribution of claims. This paper makes a pioneering attempt to model loss reserves data using quantile regression because it provides a more complete view of the causal relationships between risk factors and claim levels in loss reserving. The model is applied to two loss reserves data and results illustrate that the claim levels in different quantiles show significantly different trend patterns across lag-period and different sensitivities to initial claim level or exposure. 
Quantile regression model is further demonstrated and compared to the GT model in Chan, Choy and Makov [7] using an Israel loss reserves data. Results show that quantile regression model can capture some characteristics in the data that the sophisticated GT model has targeted for, namely the skewed error distribution due to logarithmic transformation, the shift of trend pattern for claims after a threshold pol- icy year and the extreme large and small claims. These characteristics are all allowed for in quantile regression, partly due to its nonparametric nature which avoids some model assumptions in the parametric mean regression. Forecast of total claims using a quantile level of $\tau=0.75$ is similar to the forecast using GT model. For practicing actuaries, the idea of using a sophisticated model is less attractive. This is reflected by the fact that most actuaries use solely the CL model and rarely attempt any other models. Although the performance of quantile regression model is less satisfactory than the GT model for in-sample model-fit, the former model provides a slight overestimate of total claims whereas the latter an underestimate which is less desirable because it will weaken the solvency for an insurance company and increase the risk of bankruptcy. Another practical advantage of quantile regression is that it can be easily implemented using the quantreg package in R. In conclusion, quantile regression model offers an attractive methodological advancement in forecasting loss reserves.

\section{Acknowledgment}

We gratefully acknowledge Professor Udi E. Makov, Department of Statistics, University of Haifa, Israel and Miss Alice Dong, Insurance Australia Group Limited for kindly providing the loss reserves data set from Israel and Queensland, Australia respectively to demonstrate the proposed model in this paper. This research was initiated during the visit to Professor Cathy W.S. Chen, Graduate Institute of Statistics and Actuarial Science, Feng Chia University, Taiwan.

\section{References}

[1] Barnes, M.L. and Hughes, A.W. (2002). A quantile regression analysis of the cross section of stock Market Returns, No 02-2, Working Papers from Federal Reserve Bank of Boston.

[2] Buchinsky, M. (1994). Changes in the U.S. wage structure 1963-1987: application of quantile regression, Econometrica 65, 1129-1151.

[3] Buchinsky, M. (1995). Quantile regression box-cox transformation model, and the U.S. wage structure, 1963-1987, Journal of Econometrics 65, 109-154.

[4] Buchinsky, M. (1998). Recent advances in quantile regression models: a practical guideline for empirical research, Journal of Human Resources 33, 88-126.

[5] Cade, B.S. and Noon, B.R. (2003). A gentle introduction to quantile regression for ecologists, Front Ecol. Environ. 1(8), 412-420. 
154 Predicting loss reserves using quantile regression Running title: Quantile regression loss reserve models

[6] Cade, B.S., Terrell, J.W. and Schroeder, R.L. (1999). Estimating effects of limiting factors with regression quantiles, Ecology 80, 311-323.

[7] Chan, J.S.K., Choy, S.T.B. and Makov, U.E. (2008). Dynamic and robust models for loss reserves using generalized-t distribution, ASTIN Bulletin 38, 207-230.

[8] de Jong, P. and Heller, G.Z. (2008). Generalized linear models for insurance data, Cambridge University Press, Cambridge.

[9] Eide, E. and Showalter, M.H. (1998). The effect of school quality on student per- formance: a quantile regression approach, Economic Letters 58, 345-350.

[10] Engle, R.F. and Manganelli, S. (1999) CAViaR: conditional value at risk by quantile regression, national bureau of economic research working paper no. 7341.

[11] Gutenbrunner, C., Jureckova', J., Koenker, R. and Portnoy, S. (1993). Tests of lin- ear hypotheses based on regression rank scores, Journal of Nonparametric Statistics 2, 307333.

[12] Klugman, S.A., Panjer, H.H. and Willmot, G.E. (2008). Loss Models: From Data to Decision, Third edition, John Wiley, Hoboken NJ.

[13] Koenker, R.W. and Basset, G.J. (1978). Regression quantiles, Econometrica 46, 33-50.

[14] Koenker, R.W. and Hallock, K.F. (2001). Quantile regression, Journal of Economic Perspectives 15, 143-156.

[15] Koenker, R.W. and Machado, J.A.F. (1999). Goodness of fit and related inference processes for quantile regression, J. Am. Stat. Assoc. 94, 1296-1310.

[16] Koenker, R.W. and Protnoy, S. (1997). The Gaussian Hare and the Laplacean Tortoise: computability of squared-error vs Absolute Error Estimators, (with discussion), Statistical Science 12, 279-300.

[17] Koenker, R.W. and D’Orey, V. (1987). Computing regression quantiles, Applied Statistics 36, 383-393.

[18] Koenker, R.W. and D'Orey, V. (1994). A remark on algorithm AS229: Computing dual regression quantiles and regression rank scores, Applied Statistics 43, 410-414.

[19] Kudryavtsev, A.A. (2009). Using quantile regression for rate-making, Insurance: Mathematics and Economics 45, 296-304. 
[20] Pitt, D.G.W. (2006). Regression quantile analysis of claim termination rates for income protection insurance, Annals of Actuarial Science 1(II), 345-357.

[21] Portnoy, E. (1997). Regression-quantile graduation of Australian life tables, 1946- 1992, Insurance: Mathematics and Economics 21, 163-172.

[22] Taylor, G.C. (2000). Loss Reserving - An Actuarial Perspective Kluwer Academic Publishers, Norwell, Mass.

Received March 15, 2013; accepted November 10, 2013.

Chan, J.S.K.

Department of Mathematics and Statistics Sydney University

The University of Sydney, NSW 2006, Australia.

Tel: 61283514873

jchan@maths.usyd.edu.au 
